\title{
COMPARISON OF TWO METHODS OF MULTIPLYING DISTRIBUTIONS
}

\author{
JOHAN TYSK
}

\begin{abstract}
There is no canonical way of defining a product of distributions. In the present paper we compare two different methods of defining a product of distributions. These methods are based on the sequential and functional-analytic approaches to distributions.
\end{abstract}

Introduction. In many applications of distribution theory a product of distributions occurs. There is, however, no canonical way to define such a product. In this article we shall discuss two different methods of defining a product of two distributions. The sequential approach leads to one way of defining a product, and, using Fourier analysis, we obtain a second product based on the functional-analytic definition of distributions. We call this product the product in the Fourier sense; it is essentially defined as in [1]. We shall compare the two definitions and prove that if that product exists in the Fourier sense, it also exists in the sequential sense, and the products are equal.

This article is based on an undergraduate thesis published at the Department of Mathematics at Uppsala University in September 1981. Professor Christer O. Kiselman gave me invaluable help both with my undergraduate thesis and the present paper. I take this opportunity to thank him.

The sequential method. First let us study a product based on the sequential approach. To do this we will need so-called $\delta$-sequences: Let $a_{n}, n \geqslant 1$, be positive real numbers such that $a_{n} \rightarrow 0$ as $n \rightarrow \infty$. Let $d_{n} \in C_{0}^{\infty}\left(B\left(0, a_{n}\right)\right)$ where

$$
B\left(0, a_{n}\right)=\left\{x \in \mathbf{R}^{m}:|x|<a_{n}\right\} \text {. }
$$

Assume that

$$
\int d_{n}(x) d x=1
$$

and that

$$
\int\left|d_{n}(x)\right| d x \leqslant M
$$

independently of $n$. Such a sequence will be called a $\delta$-sequence.

Received by the editors January 11, 1984.

1980 Mathematics Subject Classification. Primary 46F10; Secondary 46F12, 42B99.

Key words and phrases. Distribution, $\delta$-sequence, Fourier transform, convolution. 
Note that we only require that the $L^{1}$-norm of $d_{n}, n \geqslant 1$, is uniformly bounded. It is customary to have stronger assumptions, for instance that $d_{n}$ are all positive as in [2], or that

$$
d_{n}(x)=n^{m} d_{1}(n x), \quad n \geqslant 1 .
$$

We can now define a product of distributions.

Definition 1. We say that the product of two distributions $U$ and $V$ exists in the sequential sense in $\mathbf{R}^{m}$ if $\left(U * d_{n}\right)\left(V * e_{n}\right)$ converges in the sense of distributions in $\mathbf{R}^{m}$ for all $\delta$-sequences $\left(d_{n}\right)$ and $\left(e_{n}\right)$. Its limit is called the product of $U$ and $V$.

Note that the assumption that the limit exists for all pairs of $\delta$-sequences implies that the limit is independent of the choice of $\delta$-sequences. Definition 1 is slight modification of the product of distributions appearing in [2]: there the product is said to exist if $\left(U * d_{n}\right)\left(V * d_{n}\right)$ converges in the sense of distribution for every $\delta$-sequence $\left(d_{n}\right)$ with $d_{n} \geqslant 0, n \geqslant 1$. This definition is strictly weaker than our definition as one sees from the example

$$
\delta P V\left(\frac{1}{x}\right)=-\frac{1}{2} \frac{d \delta}{d x}
$$

where $\delta$ denotes the Dirac $\delta$-function. Using the definition in [2] this equality holds, but the product $\delta P V(1 / x)$ does not exist in the sense of Definition 1.

The Fourier method. We proceed to study a product using Fourier analysis and the functional analytic way of defining distributions. This product is essentially the product appearing in [1] and is a generalization of the product in [3] and [4, Theorem 8.2.10].

The Fourier transform of a distribution $u$ with compact support is denoted by $\hat{u}$ and defined to be the function

$$
\mathbf{R}^{m} \ni \xi \mapsto u_{x}\left(e^{-2 \pi i\langle x, \xi\rangle}\right)
$$

where the right-hand side denotes $u$ acting on the function $x \mapsto e^{-2 \pi i\langle x, \xi\rangle}$. The inverse Fourier transform of $u$, denoted by $\breve{u}$, is defined by

$$
\mathbf{R}^{m} \ni \xi \mapsto u_{x}\left(e^{2 \pi i\langle x, \xi\rangle}\right) .
$$

We are now ready to state the definition of a product of distributions based on Fourier analysis.

Definition 2. We say that the product of $U, V \in \mathscr{D}^{\prime}\left(\mathbf{R}^{m}\right)$ exists in the Fourier sense if for every $x \in \mathbf{R}^{m}$ there exists a neighborhood $\Omega_{x}$ of $x$ such that

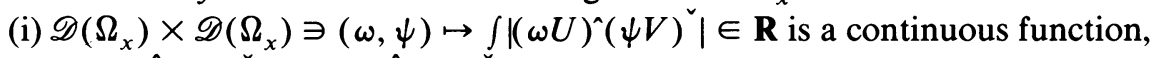

(ii) $\int(\omega U) \hat{(}(\psi V)=\int(\omega V) \hat{(} \psi(\psi)$ for $\omega, \psi \in \mathscr{D}\left(\Omega_{x}\right)$.

The action of the product $U V$ on $\omega \in \mathscr{D}\left(\Omega_{x}\right)$ is defined as follows:

$$
U V(\omega)=\int(\omega U)^{\wedge}(\psi V)^{\nu}
$$

where $\psi=1$ on supp $\omega$. 
REMARKS. The assumed continuity of the function in (i) implies that

$$
\omega \mapsto \int(\omega U)^{\wedge}(\psi V)^{\vee}
$$

is continuous as a function of $\omega \in \mathscr{D}\left(\Omega_{x}\right)$, i.e. this expression as a function of $\omega \in \mathscr{D}\left(\Omega_{x}\right)$ defines an element of $\mathscr{D}^{\prime}\left(\Omega_{x}\right)$.

By (ii) this product is independent of the choice of $\psi$. The products $U V$ in $\mathscr{D}^{\prime}\left(\Omega_{x}\right)$ for different $x$ piece together to define a product $U V$ in $\mathscr{D}^{\prime}\left(\mathbf{R}^{m}\right)$.

We also remark that the assumed continuity in (i) will be used only for very special sequences of test functions. Ambrose's definition, [1, p. 77], appears to be slightly more general than our Definition 2, his conditions (2.12) and (2.14) being combined into (i) here. However, when he uses the product in $\$ 4$ of [1], our definition would serve as well.

$A$ theorem on the relation between the products in the sequential and Fourier senses.

We can now state our theorem on the relation between these two products, which says that if the product exists in the Fourier sense it also exists in the sequential sense and the products are equal.

Theorem. Assume that the product of $U$ and $V$ exists in the Fourier sense. Then $\left(U * d_{n}\right)\left(V * e_{n}\right)$ converges in the sense of distributions where $\left(d_{n}\right)$ and $\left(e_{n}\right)$ are any $\delta$-sequences. Furthermore for every $x \in \mathbf{R}^{m}$ and every $\omega, \psi \in \mathscr{D}\left(\Omega_{x}\right)$ with $\psi=1$ on supp $\omega$ we have

$$
\int(\omega U)^{\wedge}(\psi V)^{\swarrow}=\lim _{n \rightarrow \infty} \int\left(U * d_{n}\right)\left(V * e_{n}\right) \omega .
$$

Here $\Omega_{x}$ is as in Definition 2.

For the proof of the theorem we need the following lemma.

LEMMA. Let $U, V,\left(d_{n}\right)$ and $\left(e_{n}\right)$ be as in the statement of the theorem and let $\omega, \psi \in \mathscr{D}\left(\Omega_{x}\right)$ be fixed. Then

$$
\int\left(\left(\omega\left(U * d_{n}\right)\right)^{\wedge}\left(\psi\left(U * e_{n}\right)\right)^{\nu}-\left((\omega U) * d_{n}\right)^{\wedge}\left((\psi V) * e_{n}\right)^{\nu}\right)
$$

tends to zero as $n$ tends to infinity.

Proof of Lemma. Let $n$ be fixed. We write the integrand of the lemma as

$$
\begin{aligned}
U\left(\int \omega(x) d_{n}(x-s) e^{-2 \pi i\langle x, \xi\rangle} d x\right) \\
\cdot V\left(\int \psi(y) e_{n}(y-t) e^{2 \pi i\langle y, \xi\rangle} d y\right) \\
-U\left(\int \omega(s) d_{n}(x-s) e^{-2 \pi i\langle x, \xi\rangle} d x\right) \\
\cdot V\left(\int \psi(t) e_{n}(y-t) e^{2 \pi i\langle y, \xi\rangle} d y\right)
\end{aligned}
$$


We now change the variable $x$ to $z+s$ and $y$ to $w+t$. This gives the following expression

$$
\begin{gathered}
U\left(\int \omega(z+s) d_{n}(z) e^{-2 \pi i\langle z, \xi\rangle} e^{-2 \pi i\langle s, \xi\rangle} d z\right) \\
\cdot V\left(\int \psi(w+t) e_{n}(w) e^{2 \pi i\langle w, \xi\rangle} e^{2 \pi i\langle t, \xi\rangle} d w\right) \\
-U\left(\int \omega(s) d_{n}(z) e^{-2 \pi i\langle z, \xi\rangle} e^{-2 \pi i\langle s, \xi\rangle} d z\right) \\
\cdot V\left(\int \psi(t) e_{n}(w) e^{2 \pi i\langle w, \xi\rangle} e^{2 \pi i\langle t, \xi\rangle} d w\right)
\end{gathered}
$$

Letting $U$ and $V$ act inside the integration signs this difference can be written as the sum of the following two integrals:

$$
\begin{gathered}
\int\left(d_{n}(z) e^{-2 \pi i\langle z, \xi\rangle} U_{s}(\omega(z+s)-\omega(s)) e^{-2 \pi i\langle s, \xi\rangle}\right. \\
\left.\cdot e_{n}(w) e^{2 \pi i\langle w, \xi\rangle} V_{t} \psi(w+t) e^{2 \pi i\langle t, \xi\rangle}\right) d z d w \\
+\int\left(d_{n}(z) e^{-2 \pi i\langle z, \xi\rangle} U_{s} \omega(s) e^{-2 \pi i\langle s, \xi\rangle}\right. \\
\left.\cdot e_{n}(w) e^{2 \pi i\langle w, \xi\rangle} V_{t}(\psi(w+t)-\psi(t)) e^{2 \pi i\langle t, \xi\rangle}\right) d z d w
\end{gathered}
$$

where for instance

$$
U_{s}(\omega(z+s)-\omega(s)) e^{-2 \pi i\langle s, \xi\rangle}
$$

denotes $U$ acting on the function

$$
s \mapsto(\omega(z+s)-\omega(s)) e^{-2 \pi i\langle s, \xi\rangle} .
$$

Letting

$$
f(z, w, \xi)=U_{s}(\omega(z+s)-\omega(s)) e^{-2 \pi i\langle s, \xi\rangle} V_{t} \psi(w+t) e^{2 \pi i\langle t, \xi\rangle},
$$

the first integral in the sum above can be written as

$$
\int d_{n}(z) e^{-2 \pi i\langle z, \xi\rangle} e_{n}(w) e^{2 \pi i\langle w, \xi\rangle} f(z, w, \xi) d z d w .
$$

Integrating this expression with respect to $\xi$ and then changing the order of integration we get

$$
\int d_{n}(z) e^{-2 \pi i\langle z, \xi\rangle} e_{n}(w) e^{2 \pi i\langle w, \xi\rangle} f(z, w, \xi) d \xi d z d w .
$$

This is in modulus less than

$$
\int\left|d_{n}(z) e_{n}(w) f(z, w, \xi)\right| d \xi d z d w \leqslant M^{2} \sup _{(z, w) \in H_{n}} \int|f(z, w, \xi)| d \xi
$$

where

$$
M=\max \left(\sup _{n} \int\left|d_{n}\right|, \sup _{n} \int\left|e_{n}\right|\right)
$$

and

$$
H_{n}=\operatorname{supp} d_{n} \times \operatorname{supp} e_{n}
$$


The dependence of $w(\cdot+z)$ on $z$ and $\psi(\cdot+w)$ on $w$ is obviously continuous. The assumed continuity in Definition 2(i) therefore yields that

$$
\sup _{(z, w) \in H_{n}} \int|f(z, w, \xi)| d \xi
$$

tends to

$$
\int|f(0,0, \xi)| d \xi=0
$$

as $n$ tends to infinity. The other term in the sum (1) above can be estimated similarly and the lemma is proved.

Proof of The TheOReM. Choose $\varepsilon>0$ and let $\psi=1$ on supp $\omega$. Using Parseval's identity we conclude that

$$
\int\left(U * d_{n}\right)\left(V * e_{n}\right) \omega=\int \omega\left(U * d_{n}\right) \psi\left(V * e_{n}\right)=\int\left(\omega\left(U * d_{n}\right)\right)^{\wedge}\left(\psi\left(V * e_{n}\right)\right)^{\nu}
$$

By the lemma we can choose $N_{1}$ such that $n>N_{1}$ implies

$$
\left|\int\left(\omega\left(U * d_{n}\right)\right)^{\wedge}\left(\psi\left(V * e_{n}\right)\right)^{\nu}-\left((\omega U) * d_{n}\right)^{\wedge}\left((\psi V) * e_{n}\right)^{\nu}\right|<\varepsilon / 2 .
$$

Now $\hat{d}_{n}$ and $\check{e}_{n}$ are bounded and converge to 1 so by Lebesgue's dominated convergence theorem we can choose a constant $N_{2}$ such that

$$
\left|\int(\omega U)^{\wedge}(\psi V)^{\nu}-\left((\omega U) * d_{n}\right)^{\wedge}\left((\psi V) * e_{n}\right)^{\vee}\right|<\varepsilon / 2
$$

for $n>N_{2}$. Thus $n>N=\max \left(N_{1}, N_{2}\right)$ implies

$$
\left|\int(\omega U)^{\wedge}(\psi V)^{\nu}-\left(\omega\left(U * d_{n}\right)\right)^{\wedge}\left(\psi\left(V * e_{n}\right)\right)^{\nu}\right|<\varepsilon .
$$

The choice of $\varepsilon$ being arbitrary we have proved the equality in the theorem. This equality tells us that in the sense of distributions the sequence $\left(U * d_{n}\right)\left(V * e_{n}\right)$ is convergent in $\Omega_{x}$. Since the sets $\Omega_{x}$ for different $x$ cover $\mathbf{R}^{m}$ the sequence converges in $\mathbf{R}^{m}$. This finishes the proof of the theorem.

REMARK. One can easily prove that the converse of the theorem is not true. For instance the product $g \cdot \delta$ does not exist in the Fourier sense if $g$ is a continuous function which does not agree with a function in $\hat{L}^{1}$, that is the space of Fourier transforms of functions in $L^{1}$, in any neighborhood of the origin. However, using the fact that $g$ is a continuous function it is easy to see that $\left(g * d_{n}\right)\left(\delta * e_{n}\right)$ converges in the sense of distributions, where $\left(d_{n}\right)$ and $\left(e_{n}\right)$ are arbitrary $\delta$-sequences.

\section{REFERENCES}

1. W. Ambrose, Products of distributions with values in distributions, J. Reine Angew Math. 315 (1980), 73-91.

2. P. Antosik, J. Mikusinski and R. Sikorski, Theory of distributions: The sequential approach, PWN, Warsaw, 1973.

3. L. Hörmander, Fourier integral operator. I, Acta Math. 127 (1971), 79-183.

4. , The analysis of linear partial differential operators. I, Springer-Verlag, New York, 1983.

Department of Mathematics, University of California, los Angeles, California 90024 